\title{
Discrimination of Shockable Tachycardia by Morphologic Stability of Ventricular Electrogram
}

\author{
ML Brown, S Sarkar \\ Medtronic, Inc, Minneapolis, MN, USA
}

\begin{abstract}
Most tachycardia episodes detected by implantable defibrillators as VF do not require shock therapy. Pace terminable VT represents $>80 \%$ of appropriately detected VF episodes. Rapidly conducted AF accounts for $\sim 75 \%$ of inappropriately shocked VF detections. An algorithm for discriminating $V T$ and AF from VF on the basis of morphologic stability has been devised. Morphologic similarity is determined using a template-matching algorithm. Morphologic stability was evaluated by comparing morphology of the current beat to that of a single previous beat. We compared the morphology of the current beat to that of beats preceding the current beat by 1, 4 and 7 beats. As the delay between compared beats increased, the algorithm did better at recognizing unstable morphology, but classified more non-shockable episodes as unstable. Morphologic stability can reduce inappropriate shocks for VT by $95 \%$ and inappropriate shocks for AF by $81 \%$.
\end{abstract}

\section{Introduction}

The morbidity associated with shocks delivered by implantable defibrillators has a significant impact on the lives of patients and their families. Shocks for polymorphic ventricular tachycardia and ventricular fibrillation are necessary, life-saving therapy for which the implantable defibrillator was invented. However, many shocks delivered by implantable defibrillators are either unnecessary or inappropriate. Shocks to terminate ventricular arrhythmias that could be terminated with anti-tachycardia pacing are considered unnecessary shocks. Implantable defibrillators are typically programmed to recognize ventricular rhythms with rates faster than 188 beats per minute as fast ventricular tachycardia or ventricular fibrillation and deliver shocks. In the PainFREE Rx II [1], it was shown that more than $70 \%$ of the ventricular tachycardias between 188 and 250 beats per minute were terminated with a single empiric sequence of anti-tachycardia pacing. Ventricular fibrillation, assumed to require shocks for termination, accounted for only slightly more than $10 \%$ of all ventricular tachyarrhythmias faster than $188 \mathrm{bpm}$ [2]. Shocks are considered inappropriate when delivered by the implantable defibrillator because of inappropriate detection of supraventricular tachycardia as ventricular. Nearly $75 \%$ of the inappropriate detections resulting in inappropriate shocks are because of the over-detection of rapidly conducted atrial fibrillation. The ventricular rate during these episodes of atrial fibrillation are often faster than 200 beats per minute, a rate for which shocks are typically programmed. Though implantable defibrillators have detection criteria to discriminate AF from VT/VF, these criteria often are less effective at these high rates and the demand for high sensitivity by defibrillator detection algorithms requires that therapy be delivered when detection criteria are ambiguous. These criteria typically include comparison of atrial and ventricular rates, regularity of the ventricular rate, association of atrial and ventricular electrograms and ventricular electrogram morphology.

Aberrant conduction during rapidly conducted atrial fibrillation often results in QRS morphology different than that found during sinus rhythm. Thus, template matching algorithms that compare the QRS during ventricular high rate episodes to a baseline rhythm QRS are unable to reject because of the change in morphology. However, it is common for the aberrant QRS morphology to be relatively stable from beat to beat such that a morphology algorithm that compared adjacent or nearly adjacent beat morphologies to recognize stable morphology could be used to discriminate atrial fibrillation from ventricular fibrillation which has an inherently unstable ventricular morphology. This study was designed to evaluate algorithms that discriminate shockable from non-shockable rhythms on the basis of morphologic stability.

The top panel of figure 1 shows the ventricular electrogram during a monomorphic ventricular tachycardia. The lower panel shows the electrograms of the QRSs of the upper panel superimposed on one another to demonstrate the consistent stable morphology of ventricular tachycardia. A plot of the electrogram during atrial fibrillation is very similar though the intervals between QRSs is less regular. 


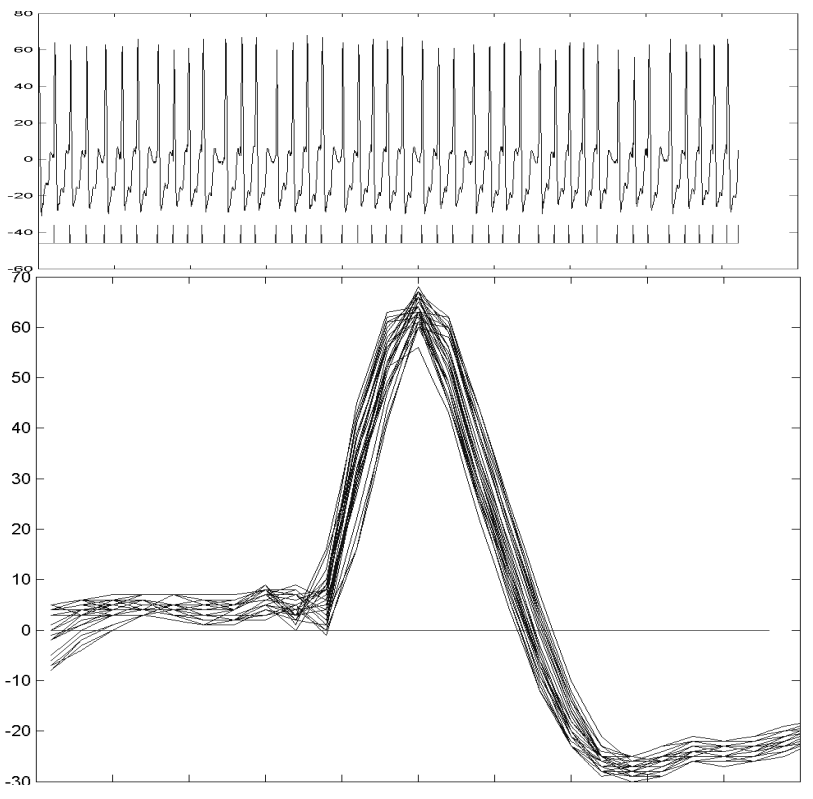

Figure 1: Top panel shows ventricular electrogram of ventricular tachycardia. The bottom panel shows electrograms of QRSs of the above panel superimposed on one another to demonstrate the stability of the QRS morphology during monomorphic VT.

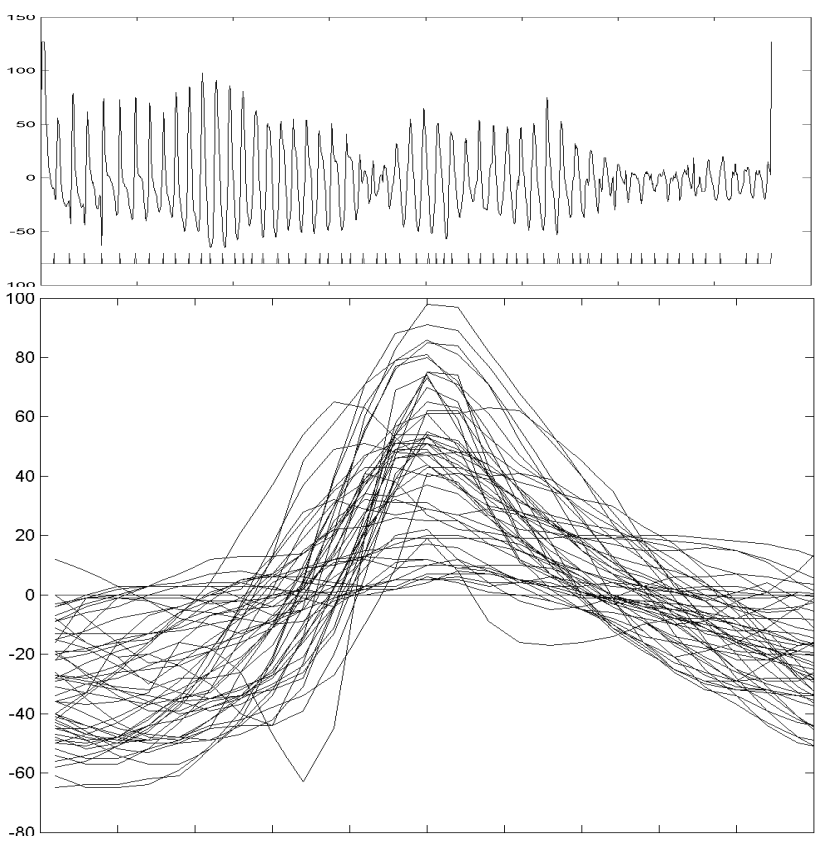

Figure 2: Top panel shows ventricular electrogram of ventricular fibrillation. The bottom panel shows electrograms of QRSs of the above panel superimposed on one another to demonstrate the instability of the QRS morphology during VF.

The top panel of figure 2 shows the ventricular electrogram during a ventricular fibrillation. The lower panel shows the electrograms of the QRSs of the upper panel superimposed on one another to demonstrate the unstable morphology of ventricular fibrillation. In this study, an algorithm to reliably discriminate the difference in morphologic stability between figures 1 and 2 was sought.

Thus, the objective of this study was to develop an algorithm based on the stability of the QRS morphology to discriminate non-shockable (AF and VT) from shockable (polymorphic VT and VF) rhythms. The algorithm was to use techniques that are amenable to implementation in an implantable defibrillator. The candidate algorithms were evaluated using stored episode data from implantable defibrillators.

\section{Methods}

Data used for this analysis were implantable defibrillator stored episode data from the GEM DR dual chamber ICD. Episodes that sustained at least 18 beats with all ventricular intervals less than $340 \mathrm{~ms}$ were included if they stored an RV coil-can electrogram and were at least 10 minutes separated from the most recent device therapy. The database consisted of $55 \mathrm{VF} / \mathrm{PVT}$ episodes (21 patients), 104 MVT episodes (44 patients) and 63 AF episodes (29 patients). The algorithms were developed on a subset of the data and subsequently evaluated on the entire dataset. Ventricular arrhythmia episodes were classified as non-shockable if they had regular intervals, and stable QRS morphology and amplitude. Otherwise they were classified as shockable.

Morphologic similarity of beats was assessed using the same wavelet transform method found in the Marquis VR ICD. Figure 3 illustrates how the wavelet algorithm works. On the left is shown a $200 \mathrm{~ms}$ segment of the RV coil-can electrogram centered on the sensed ventricular event. The electrogram is sampled at $250 \mathrm{~Hz}$. The second vertical panel shows the wavelet functions used to analyze electrogram morphology. Each function has the same general shape, but varies according to scale and time shift. The QRS is correlated with each of these wavelet functions to produce a coefficient or multiplier for each function. The coefficient is large for those functions that are most similar to the QRS and small for those which are dissimilar. The coefficients are sorted according to magnitude and only the largest are used to represent the QRS. The coefficients are shown graphically in the first vertical panel. The original QRS can be reconstructed from the coefficients by multiplying the coefficient by its associated wavelet function and adding the results. As the signal is reconstructed one coefficient at a time, one can see that a good representation requires only a subset of the coefficients. Thus, the QRS can be adequately represented by a subset 
of the coefficients which provides economy of storage and computational burden.

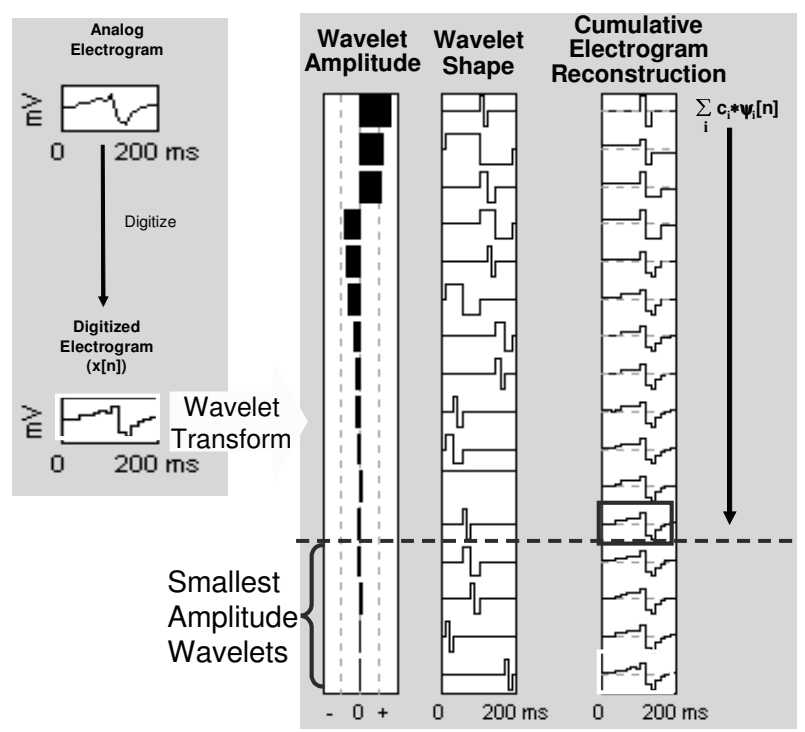

Figure 3: The panel in the upper left shows the electrogram of a QRS that is to be analyzed. Once it is digitize, the correlation between the QRS and an analysis wavelet is determined; this is the wavelet transform. The panel on the right shows the reconstruction of the original QRS by adding together the weighted analyzing wavelets rank ordered by their magnitude.

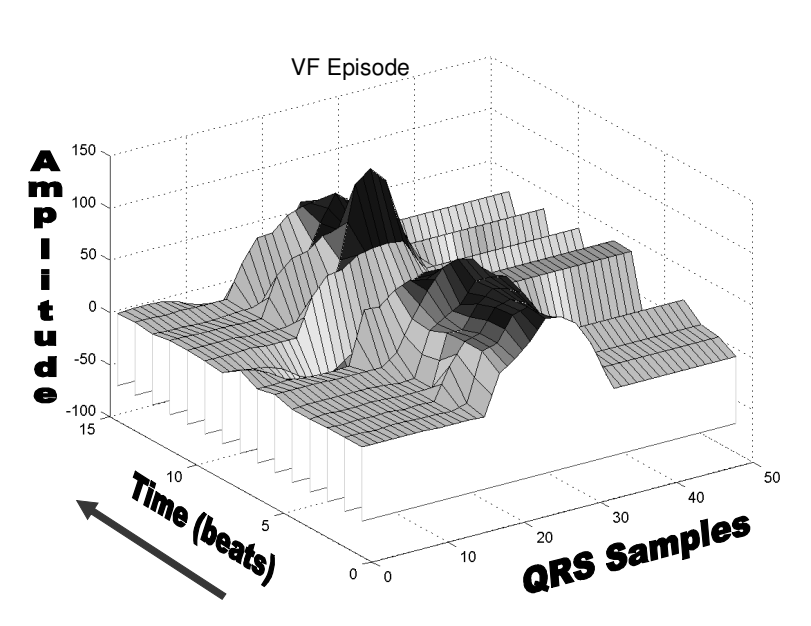

Figure 4: This graph shows successive beats of a VF episode. Notice how successive beats of VF look similar while beats that are more distant are dissimilar.

The initial algorithm method we tested compared the morphology of adjacent beats to determine morphologic stability. We determined that VF appears to be quite stable when comparing adjacent beats. Figure 4 shows successive beats of a VF episode. Notice that adjacent beats are often quite similar, but beats separated by a few beats are more dissimilar. We adjusted our algorithm to evaluate the similarity of beats separated by differing amounts and to evaluate what separation provided the best overall discrimination of shockable and nonshockable rhythms.

The algorithm is depicted in figure 5. The morphology stability algorithm compares the morphology of the current beat to the morphology of a template created from the Nth previous beat, where we tested delays of 1-7 beats. If 4 of the most recent 8 comparisons are MATCHES, then the morphology is stable and the rhythm is declared non-shockable; otherwise the rhythm is declared shockable. When used in an implantable defibrillator, the therapy delivered at the time of initial detection would determined by the most recent rhythm decision - stable or unstable.

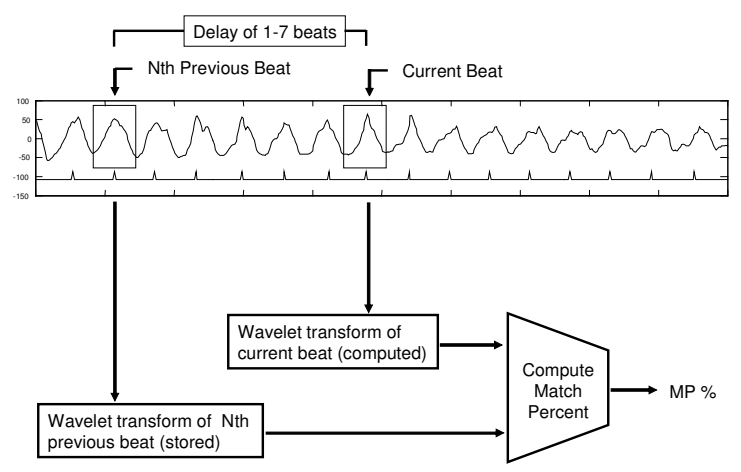

If $\geq 4$ of 8 comparisons $=\mathrm{MATCH}$ then classify rhythm as VT

Figure 5: The morphology stability algorithm compares the morphology of the current beat to the morphology of a template created from the Nth previous beat, where we tested delays of 1-7 beats.

\section{Results}

Figure 6 shows how, on the average, the match percentage varies with the delay between beats compared for different rhythms. The horizontal axis is the delay between beats that are compared and the vertical axis is the average match percentage for all beats in a single episode. The three lines show the average match percentage as a function of distance between beats for a VF episode, a VT episode and an AF episode. Notice that the average match percentage stays relatively constant for $\mathrm{VT}$ and AF as the delay between beats increases because the morphology is a very stable. For VF, as the delay increases, the average match percentage decreases because the morphology is unstable.

In figure 7 , the results for 3 values of delay are displayed: 1,4 and 7 beats. The dark gray bars represent $\mathrm{VF}$ episodes, the light gray bars $\mathrm{AF}$ episodes and the black bars VT episodes. The bars are $100 \%$ in height. If a bar is completely above the line, such as the VF bar in 
the ideal example, then all episodes are detected as VF. If a bar is completely below the line such as the VT bar in the ideal example, then all episodes are detected as VT. The numbers in the box are the numbers of episodes detected as VT (Below the line) and VF (above the line). The left-most graph shows the results for a 1 beat delay. Only a little more than half of the VF episodes were classified as VF while nearly all VT episodes were correctly classified. As the delay increases to 4 and 7 , more VF, VT and AF episodes are classified as VF. A delay of 4 produces the highest percentage of correct classifications overall while a delay of 7 gives the highest percentage of VFs correctly classified.

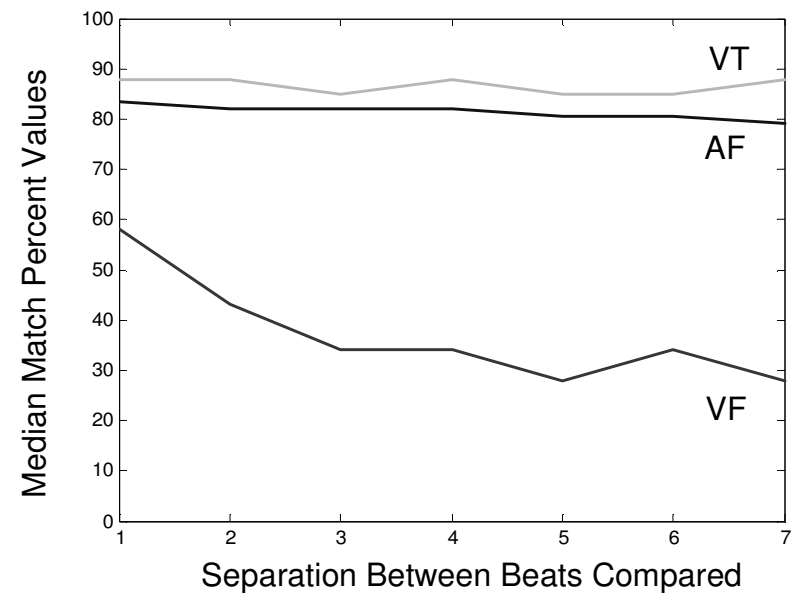

Figure 6: The horizontal axis is the delay between beats that are compared and the vertical axis is the average match percentage for all beats in a single episode.

\section{Discussion and conclusions}

The morbidity associated with shocks delivered by implantable defibrillators has a significant impact on the lives of patients and their families. Many unnecessary or inappropriate shocks can be prevented by an algorithm to discriminate shockable from non-shockable rhythms. We investigated a morphology stability algorithm that could be used for this purpose. It was able to achieve $91 \%$ (145/159) correct classification of VT/VF episodes. It can reduce shocks for fast VT by $95 \%$ (99/104) while delivering first therapy shocks to $84 \%$ (46/55) of VF episodes. It can reduce inappropriate detection of $\mathrm{AF}$ by $81 \%$ (51/63). The algorithm is feasible for implementation in an ICD because it uses methods already incorporated in market released devices.

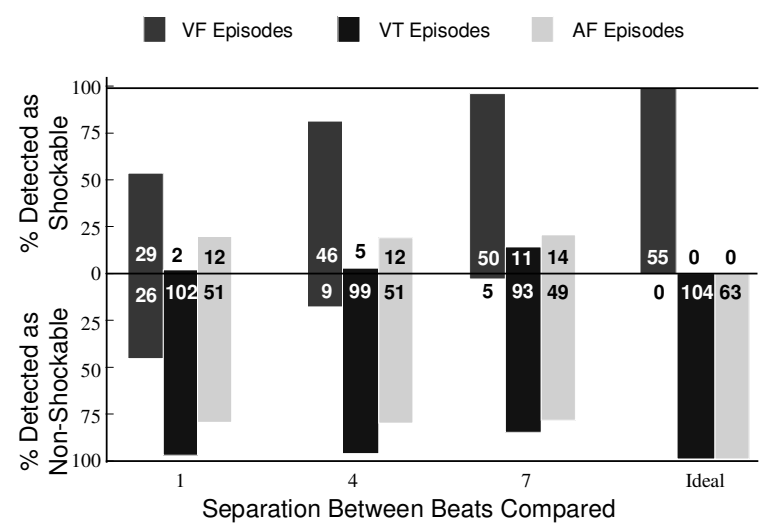

Figure 7: Results for 3 values of delay are displayed: 1, 4 and 7 beats. The bars are $100 \%$ in height. The numbers in the box are the numbers of episodes detected as VT (below the line) and VF (above the line).

\section{Acknowledgements}

The authors would like to thank Jeff Gillberg, Jian Cao and Rob Stadler for their feedback and assistance with this work.

\section{References}

[1] Wathen MS, et. al. Prospective Randomized Multicenter Trial of Empirical Antitachycardia Pacing Versus Shocks for Spontaneous Rapid Ventricular Tachycardia in Patients With Implantable Cardioverter-Defibrillators, Circulation 2004;110:2591-2596.

[2] Sweeney MO, et. al. Appropriate and Inappropriate Ventricular Therapies, Quality of Life, and Mortality Among Primary and Secondary Prevention Implantable Cardioverter Defibrillator Patients, Circulation 2005; 111:2898-2905.

[3] Wilkoff BL, et. al. Critical Analysis of Dual-Chamber Implantable Cardioverter-Defibrillator Arrhythmia Detection: Results and Technical Consideration. Circulation 2001;103:381-386.

[4] Swerdlow CD, et. al. Discrimination of Ventricular Tachycardia from Supraventricular Tachycardia by a Downloaded Wavelet-Transform Morphology Algorithm. J Cardiovasc Electrophysiol 2002; 13:432-441.

[5] Brown, ML, Sarkar S. Discrimination of VT and VF by Stability of Ventricular Electrogram Morphology. Pacing and Clinical Electrophysiology 2003; 26:988.

Address for correspondence:

Mark L. Brown

Medtronic

7000 Central Ave NE

Mail Stop CW330

Minneapolis, MN 55432

Mark.L.Brown@medtronic.com 